\title{
COMPRESSIVE DYSPHAGIA SECONDARY TO PARAESOPHAGEAL GIST TUMOR: AN UNUSUAL CAOL:
}

\section{Calvo Bernal MM, Lázaro Sáez M, Moreno Moraleda I}

Complejo Hospitalario Torrecárdenas. Almería.

Resumen

Los tumores del estroma gastrointestinal (GIST) son neoplasias muy poco frecuentes del tubo digestivo. El estómago y el intestino delgado son los lugares de presentación más comunes representando aproximadamente el $85 \%$ de los casos siendo la ubicación esofágica excepcional. Presentamos el caso de un varón de 76 años que presenta disfagia a sólidos, asociado a vómitos alimenticios postprandiales y pérdida ponderal de 3 meses de evolución que es diagnosticado de un tumor GIST esofágico.

Palabras clave: disfagia, tumor esofágico, tumor del estroma gastrointestinal.

\section{CORRESPONDENCIA}

María del Mar Calvo Bernal

Complejo Hospitalario Torrecárdenas

04009 Almería

marcalvobernal@gmail.com

Fecha de envio: 08/04/2021

Fecha de aceptación: 01/08/2021
Abstract

Gastrointestinal stromal tumors (GIST) are very infrecuent neoplasms of the digestive tract. The stomach and the small bowel are the most frecuent locations, constituting $85 \%$ of the cases. Esophageal location is exceptional. We present the case of a 76 years old male who has dysphagia to solids asociated to postprandial alimentary vomits and weight loss for three months who is diagnosed of an esophageal GIST.

Keywords: dysphagia, esophageal tumor, gastrointestinal stromal tumor.

\section{Introducción}

Los tumores del estroma gastrointestinal (GIST) representan menos del $2 \%$ de las neoplasias del tubo digestivo, siendo sin embargo, las neoplasias mesenquimales del tracto gastrointestinal más frecuentes ${ }^{1}$. Pueden localizarse en cualquier parte del tracto gastrointestinal. Los órganos más comprometidos son estómago (50-60\%) seguido del intestino delgado (20-30\%), siendo el GIST esofágico muy poco común, con menos de $1 \%$ de incidencia. 
Su incidencia es de 4-10 casos/millón de habitantes/año, siendo más frecuentes entre la cuarta y la sexta década de la vida, con un leve predominio en el sexo masculino. Presentan unos rasgos inmunohistoquímicos característicos que le han permitido constituir una entidad propia y diferenciarlos de otras neoplasias del tubo digestivo.

\section{Caso Clínico}

Presentamos el caso de un varón de 76 años con antecedentes de enfermedad pulmonar obstructiva crónica severa y claudicación intermitente secundaria a oclusión vascular, que es derivado a Consultas Externas de Aparato Digestivo por presentar disfagia a sólidos de 3 meses de evolución, asociado a vómitos alimenticios postprandiales y pérdida ponderal de 5 kilos en último mes sin otra sintomatología asociada.

A la exploración, presenta abdomen blando y depresible, distendido y no doloroso a la palpación. En la analítica, destaca anemia normocítica (hemoglobina 10,4 gr/dl).

Se realiza una endoscopia digestiva alta en la que se objetiva esofagitis péptica grado II/IV sin otros hallazgos destacables así como una radiografía de tórax y tomografía computarizada(TC) toracoabdominal (Figuras 1 y 2), en el que se aprecia a nivel torácico una masa paraesofágica izquierda de $6 \times 4,6 \mathrm{~cm}$ con contenido heterogéneo, bien delimitada, y un tránsito esófago-gastro-duodenal (Figura 3), en el que se observa esófago de morfología, calibre y peristaltismo normales, con patrón mucoso normal sin observar estenosis esofágica

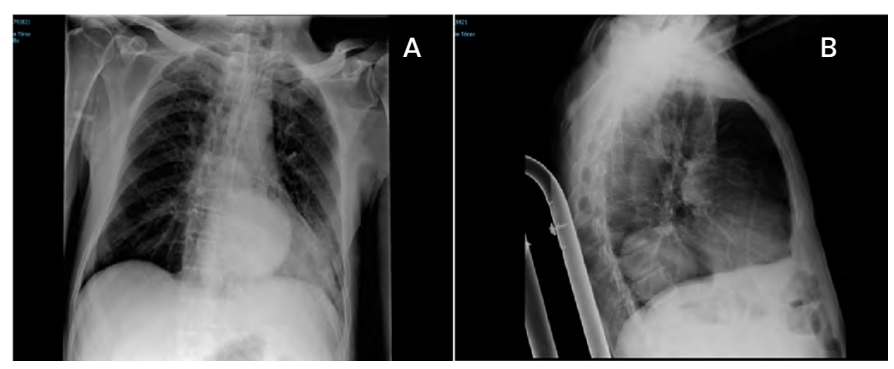

Figura 1

Radiografía de tórax. A. Posteroanterior. Se aprecia masa paraesofágica izquierda superpuesta a silueta cardiaca, de bordes redondeados, bien definidos. B.Lateral. Se observa dicha masa en proyección lateral, retrocardiaca, que ocupa más de un tercio de hemitórax izquierdo.
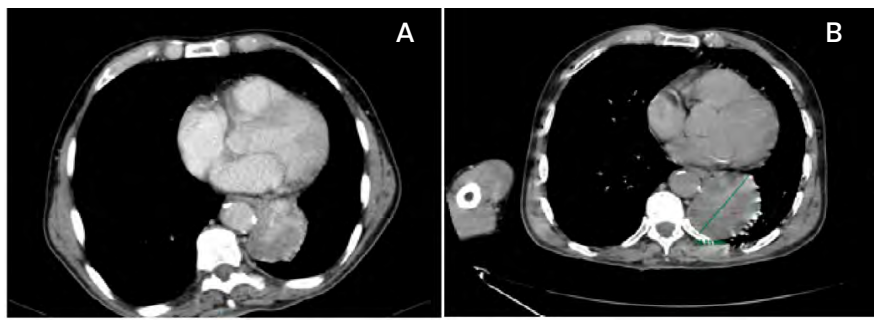

Figura 2

TC toracoabdominal. A. Al diagnóstico. Corte sagital en el que se aprecia a nivel torácico una masa paraesofágica izquierda de $6 \times 4,6 \mathrm{~cm}$, de contenido heterogéneo aunque bien delimitada. B. Al año de evolución. Se observa crecimiento de masa tumoral con respecto a estudio previo, asociado a compresión de lóbulo pulmonar inferior izquierdo y derrame pleural ipsilateral.

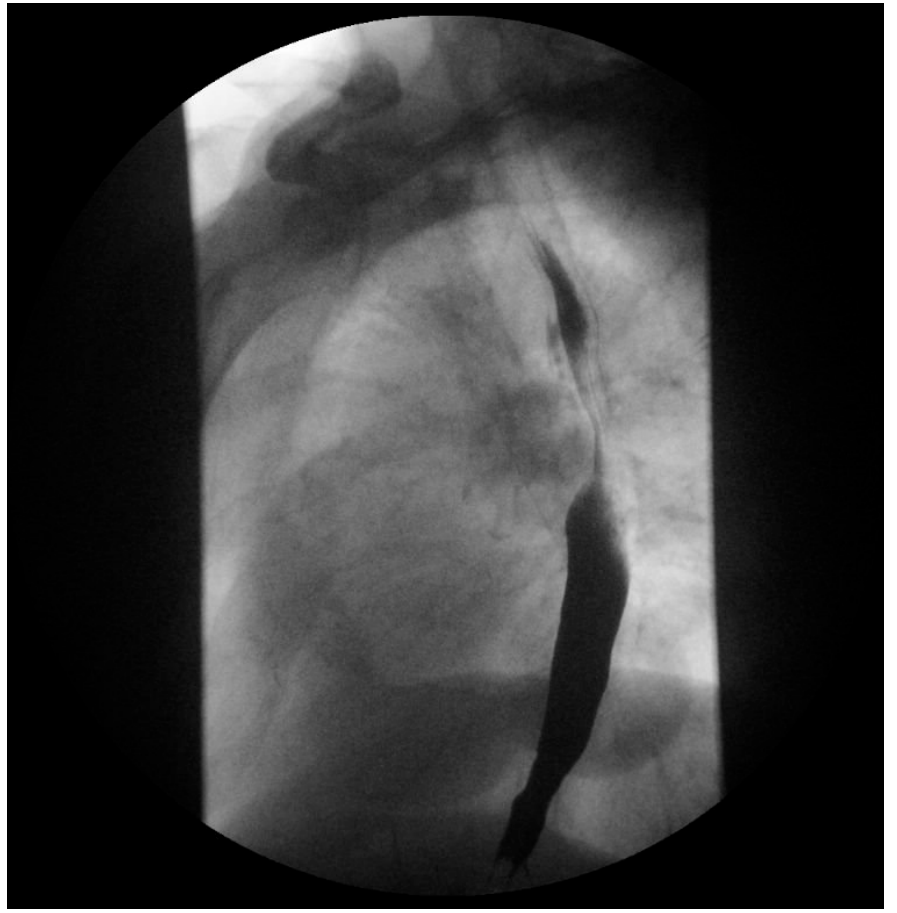

Figura 3

Tránsito esófago-gastro-duodenal. Se observa esófago de morfología, calibre y peristaltismo normales, con patrón mucoso normal, sin apreciar estenosis.

secundaria a la masa previamente identificada en en TC.

Tras estos hallazgos, se realiza una ecoendoscopia que objetiva una lesión subepitelial del esófago distal heterogénea, de tipo mesenquimal con origen en la muscular propia. La citología mediante punción aspiración con aguja fina (PAAF) guiada por ecoendoscopia muestra un patrón fusocelular, sin áreas de necrosis, altamente positivo en la inmunohistoquímica para el marcador c-KIT, compatible con tumor del estroma gastrointestinal esofágico.

Finalmente se presenta el caso en sesión conjunta con Cirugía General y ante la mejoría clínica, teniendo en cuenta la comorbilidad del paciente y la agresividad de la cirugía, se consensúa actitud conservadora en ese momento, consiguiéndose un adecuado control de la sintomatología con tratamiento médico y remitiéndose a consulta de Oncología médica.

Se continúa seguimiento en consulta, presentando en última visita empeoramiento de la clínica, con aumento de episodios eméticos diarios, por lo que se realiza nuevo TC en el que se aprecia crecimiento de la masa tumoral, asociado a compresión del lóbulo pulmonar inferior izquierdo y derrame pleural. El paciente es remitido de nuevo a Consulta de Cirugía General, en espera de nueva valoración de intervención quirúrgica por su parte.

\section{Discusión}

Los tumores del estroma gastrointestinal (GIST) son muy poco frecuentes. Tienen una mayor prevalencia en pacientes con neurofibromatosis tipo I. La inmunohistoquímica de los tumores del estroma gastrointestinal es fundamental para su diagnóstico. La 
mayoría presentan una mutación con ganancia de función en el gen c-KIT con actividad tirosina-quinasa definiendo una proliferación celular no regulada, aunque la negatividad de este marcador en presencia de un patrón histológico sugerente no descarta el diagnóstico.

Los GIST esofágicos son más comunes en el tercio medio a distal del esófago. Suelen ser pequeños y asintomáticos, pero en ocasiones pueden alcanzar un gran tamaño y presentarse con disfagia, odinofagia, hemorragia digestiva alta por ulceración mucosa, o intraperitoneal por rotura tumoral, dolor abdominal, anemia, pérdida de peso o náuseas.

Alrededor de un $50 \%$ de los tumores del estroma gastrointestinal están localizados en el momento de su diagnóstico. Su diseminación metastásica más frecuente es por vía hematógena hacia el hígado, siendo excepcional la diseminación linfática.

El diagnóstico diferencial se debe realizar fundamentalmente con el leiomioma, leiomiosarcoma, schwanoma, neurofibroma y tumores neuroendocrinos como el carcinoide. La ecoendoscopia es una prueba crucial para el diagnóstico de tumores subepiteliales, proporcionando información sobre la capa de la pared en la que se origina, la naturaleza de la lesión y su tamaño y permite realizar una aspiración con aguja fina que proporcione un diagnóstico concluyente.

El tratamiento depende de la ubicación y el tamaño del tumor, la extensión de la diseminación y la presentación clínica. Los GIST mayores de $2 \mathrm{~cm}$, aquellos que presenten manifestaciones clínicas asociadas o un patrón sugerente de malignidad en la ecoendoscopia (borde irregular, espacios quísticos, ulceración, focos ecogénicos y heterogeneidad) deben ser resecados ${ }^{2}$. Para el resto de casos, se debe llevar a cabo un seguimiento endoscópico periódico.

La cirugía consiste en la resección completa con márgenes negativos, sin linfadenectomía asociada, salvo si existe evidencia de afectación ganglionar grosera. El tratamiento de los tumores GIST en la unión gastroesofágica (GE) es problemático ya que puede necesitar esofagectomía con gastrectomía proximal. A nivel esofágico, no existe una capa serosa que los cubra, de modo que el riesgo de rotura es extremadamente alto y la resección esofágica es una técnica que comporta alta morbimortalidad, pudiendo ocasionar deformidad o estenosis en la entrada gástrica, lo que conlleva mayores complicaciones y merma en la calidad de vida ${ }^{3}$.

Las técnicas híbridas que combinan enfoques laparoscópicos y endoscópicos pueden mejorar los resultados del paciente $\mathrm{y}$ proporcionar mejores resultados ${ }^{4}$. Cuando la resección es completa, las tasas de supervivencia están en torno al $40-55 \%$ a los cinco años. El pronóstico depende del tamaño y la tasa mitótica, la localización y la integridad de la resección ${ }^{5}$.

Se debe considerar la terapia sistémica con imatinib, un inhibidor de tirosina quinasa de molécula pequeña ${ }^{6}$. Éste puede administrarse de forma neoadyuvante en tumores límite previa resección quirúrgica, de forma adyuvante en aquellos que no lograron resección completa y presenten alto riesgo de recidiva o en aquellos no candidatos a cirugía o con enfermedad avanzada. En este caso, debe administrarse de por vida.
Como conclusión, en esta patología es fundamental un diagnóstico y resección quirúrgica precoz, lo que conllevaría un mejor pronóstico. El enfoque terapéutico debe ser personalizado para cada paciente, teniendo en cuenta la comorbilidad así como el tamaño y localización tumoral y decidir de forma multidisciplinar la terapéutica que suponga un menor impacto en la calidad de vida de los pacientes.

\section{Bibliografía}

1. Theiss L, Contreras CM. Gastrointestinal Stromal Tumors of the Stomach and Esophagus. Surg Clin North Am. 2019 Jun;99(3):543-553. doi: 10.1016/j. suc.2019.02.012. PMID: 31047041

2. Akahoshi K. , Oya M. , Koga T. , Shiratsuchi Y. Manejo clínico actual del tumor del estroma gastrointestinal, World J. Gastroenterol. , 24 ( 26 ), 2018

3. Xu, X., Chen, K., Zhou, W. et al.Laparoscopic Transgastric Resection of Gastric Submucosal Tumors Located Near the Esophagogastric Junction. J Gastrointest Surg 17, 1570-1575 (2013). https://doi.org/10.1007/s11605-013-2241-2

4. Aguayo WG, Rojas CL, Molina GA, Cárdenas BA, Parreño EF, Melendez SD, Alvarez MP, Basantes VM, Aguayo JJ, Gualotuña FV. A hybrid approach for GISTs near the esophagogastric junction, a case report. Ann Med Surg (Lond). 2021 Jan 20;62:288-292. doi: 10.1016/j.amsu.2021.01.022. PMID: 33537145; PMCID: PMC7841213.

5. Lim KT. Surgical treatment of gastrointestinal stromal tumors of the stomach: current status and future perspective. Transl Gastroenterol Hepatol. 2017 Dec 7;2:104. doi: 10.21037/tgh.2017.12.01. PMID: 29354761; PMCID: PMC5762995.

6. Sharma NR, Gopakumar H. , Harrison S. , Ehmke N. , Zelt C. Tumores del estroma gastrointestinal gástrico (GIST): una serie de casos y el estado actual del arte en el diagnóstico y tratamiento de esta rara enfermedad. Gastrointest. Canc. , 50 ( 3 ) (2017) , págs. 548 - 555 , 10.1007 / s12029-017-0034-7. 